\title{
PRESIÓN INTRAOCULAR POST-TRATAMIENTO DE LA OFTALMOPATÍA TIROIDEA
}

\section{INTRAOCULAR PRESSURE AFTER TREATMENT FOR THYROID- ASSOCIATED OPHTHALMOPATY}

\author{
CRESPÍ J ${ }^{1}$, RODRÍGUEZ F ${ }^{2}$, BUIL JA ${ }^{3}$
}

\section{RESUMEN}

Objetivo: Evaluar los cambios en la presión intraocular (PIO) en pacientes afectos de oftalmopatía tiroidea (OT) tratados mediante descompresión orbitaria, cirugía sobre músculos extraoculares, o pulsos endovenosos de 6-metil-prednisolona.

Material y métodos: Se han analizado los datos de 31 pacientes que fueron tratados en nuestro Servicio entre 1998-2004. Las variables utilizadas han sido: edad, sexo, diagnóstico y tratamiento de glaucoma, tratamiento esteroides sistémicos, tipo de operación, PIO pre y postoperatoria en posición primaria de mirada (PPM) y posición en mirada hacia arriba (PMA).

Resultados: En los 20 ojos en que se realizó descompresión orbitaria, la PIO media preoperatoria en PPM fue de 17,35 (DE: 3,86) $\mathrm{mmHg}$ y de 22,45 (DE: 6,36) mmHg en PMA. La PIO postoperatoria en PPM fue de 14,24 (DE: 3,43) mmHg y de 18,20 (DE: 4,74) mmHg en PMA. En los 10 ojos de pacientes intervenidos de estrabismo, la PIO preoperatoria en PPM fue de 18,9 (DE: 3,07) $\mathrm{mmHg}$ y de 22,4 (DE: 6,14) $\mathrm{mmHg}$ en elevación. La PIO postoperatoria en PPM fue de 16,6 (DE: 3,50) y de 18,6 (DE: 3,33) mmHg en elevación. En los 12 pacientes, 24 ojos, que recibieron pulsos la PIO en PPM era de 21,33 (DE: 6,42) mmHg y de 24,45

\section{ABSTRACT}

Objective: To evaluate the change in the intraocular pressure (IOP) in subjects with thyroid-associated orbitopathy (TAO) treated with orbital decompression, extraocular muscle surgery or methylprednisolone pulse therapy.

Methods: The charts of 31 subjects with TAO who visited in our department between 1998 and 2004 were analyzed. Subject age, gender, diagnosis and treatment for glaucoma, use of systemic steroids, procedure performed and pre-operative and postoperative IOP in the primary position and in upgaze, were all evaluated.

Results: Of the 20 eyes that underwent orbital decompression, the mean pre-operative IOP was 17.35 (3.86 SD) $\mathrm{mmHg}$ and 22.45 (6.36 SD) $\mathrm{mmHg}$ in upgaze. The post-operative IOP was 14.24 (3.43 SD) $\mathrm{mmHg}$ and 18.20 (4.74 SD) $\mathrm{mmHg}$ in upgaze. The mean pre-operative IOP in the 10 eyes who had strabismus surgery was 18.9 (3.07 $\mathrm{SD}) \mathrm{mmHg}$ and 22.4 (6.14 SD) $\mathrm{mmHg}$ in upgaze. The post-operative IOP was 16.6 (3.50 SD) $\mathrm{mmHg}$ and 18.6 (3.33 $\mathrm{SD}) \mathrm{mmHg}$ in upgaze. In the 12 patients treated with methylprednisolone pulse therapy, the mean pre-treatment IOP was $21.33(6.42 \mathrm{SD}) \mathrm{mmHg}$ and $24.45(8.15 \mathrm{SD}) \mathrm{mmHg}$ in upgaze. After the treatment the mean IOP was $17.33(4.38 \mathrm{SD}) \mathrm{mmHg}$

\footnotetext{
Recibido: 31/5/06. Aceptado: 20/9/07.

Hospital de la Santa Creu i Sant Pau. Servicio de Oftalmología. Barcelona. España.

${ }^{1}$ Licenciado en Medicina. Residente de Oftalmología.

2 Licenciado en Medicina. Adjunto del Servicio de Oftalmología.

3 Doctor en Medicina. Jefe del Servicio.

Correspondencia:

Jaume Crespí

C/. Castellnou, 42, 2-1

08017 Barcelona

España

E-mail: 38685jcv@comb.es
} 
(DE: 8,15) mmHg en elevación. Después del tratamiento, la PIO en PPM fue de 17,33 (DE: 4,38) mmHg y de 20,08 (DE: 4,86) mmHg en PMA. En los pacientes con una PIO preoperatoria $\geq 21$ $\mathrm{mmHg}$ o en tratamiento para glaucoma se ha observado un descenso más marcado de la PIO ( $\mathrm{p}<$ 0,005).

Conclusiones: Se ha observado un descenso significativo de la PIO en los tres grupos de pacientes postratamiento de la OT.

Palabras clave: Oftalmopatía tiroidea, PIO, glaucoma, descompresión orbitaria, tratamiento con pulsos metilprednisolona.

\section{INTRODUCCIÓN}

La enfermedad tiroidea puede causar múltiples problemas oftalmológicos, que engloban la denominada oftalmopatía tiroidea (OT). Entre ellos se encuentra el glaucoma o la hipertensión ocular (HTO) (1) aunque su etiopatogenia aún se desconoce. Se han postulado diversas teorías: la contracción de los músculos extraoculares infiltrados y fibrosados contra el globo ocular, el aumento de la presión venosa episcleral secundaria a la congestión de la órbita o el aumento de la presión retrobulbar podrían influir en el aumento de la presión intraocular (PIO). (2)

El propósito de este estudio consiste en evaluar los cambios en la PIO en pacientes con OT que requirieron tratamiento y determinar cómo influye éste en la evolución de aquellos pacientes con glaucoma o HTO.

\section{SUJETOS, MATERIAL Y MÉTODOS}

Se han analizado los datos de pacientes diagnosticados de OT en el Servicio de Oftalmología de nuestro hospital entre el 1 de enero de 1998 y el 31 de diciembre de 2004. Se ha realizado una revisión retrospectiva de 31 pacientes consecutivos tratados mediante cirugía de descompresión orbitaria, cirugía sobre músculos extraoculares (MOE) o pulsos endovenosos de 6-metilprednisolona (6-MPDN). Los pacientes en los que no se pudieron recoger todas las variables fueron excluidos del estudio, así como otros dos pacientes por presentar pseudoexfolación. La media de edad de todo el grupo es de 48 and 20.08 (4.86 SD) $\mathrm{mmHg}$ in upgaze. In subjects with a pre-operative IOP $\geq 21 \mathrm{mmHg}$ or in treatment for glaucoma we observed a greater reduction in $\operatorname{IOP}(\mathrm{p}<0.005)$.

Conclusions: We have observed a significant reduction in IOP in the three groups of patients after treatment for TAO, however no significant difference was found between the methods used (Arch Soc Esp Oftalmol 2007; 82: 691-696).

Key words: Thyroid-associated orbitopathy, IOP, glaucoma, orbital decompression, methylprednisolone pulse therapy.

años (rango: 28-73). Un $83 \%$ de los pacientes eran mujeres (26 mujeres y 5 hombres). Estos datos, así como la indicación del tratamiento se resumen en la tabla I.

En total se obtuvieron datos de 54 ojos de 31 pacientes para su análisis. De estos pacientes, diez fueron operados de cirugía de descompresión (20 ojos), nueve fueron operados cirugía sobre MOE (diez ojos: ocho retroinserción del recto inferior y dos retroinserción de recto inferior y recto medial) y doce recibieron pulsos de corticoides (24 ojos). La cirugía de descompresión consistió en todos los pacientes en una descompresión de tres paredes orbitarias con abordaje bicoronal. En la cirugía sobre MOE se realizó una retroinserción del músculo recto inferior combinada a veces con una retroinserción del músculo recto medial. En los pacientes que recibieron pulsos de corticoides las dosis fueron: $500 \mathrm{mg}$ de 6-MPDN endovenosos 2 veces al día durante tres días. Posteriormente se administraba una pauta descendente de corticoides vía oral, empezando por $40 \mathrm{mg}$ y disminuyendo 10 mg cada semana.

La PIO preoperatoria se registró 2 semanas antes, y la postoperatoria entre 1 y 2 meses después. En los pacientes que recibieron pulsos se registró entre los 2 y 4 meses. La PIO se midió con tonómetro de aplanación de Goldmann y fue realizada siempre por el mismo oftalmólogo. Se consideró HTO valores de $\mathrm{PIO} \geq 21 \mathrm{mmHg}$ en al menos dos ocasiones. En los pacientes con diagnóstico previo de glaucoma éste se definió como un aumento de la PIO y/o una progresión documentada de la excavación papilar o una alteración del campo visual en concordancia con un adelgazamiento del anillo neurorretinia- 
Tabla I.

\begin{tabular}{lccc}
\hline & Descompresión & Cirugía MOE & Pulsos 6-MPDN \\
\hline Edad (años) m DE (r) & 43,4 DE 5,44 (35-55) & 49,9 DE $1,06(37-61)$ & 52,5 DE 3,66 (28-73) \\
Sexo mujer/hombre (n) & $10 / 0$ & $7 / 2$ & $9 / 3$ \\
Indicación tratamiento (n) & $\varnothing$ & 9 & $\varnothing$ \\
Diplopia & 7 & $\varnothing$ & $\emptyset$ \\
Proptosis & 3 & $\varnothing$ & 1 \\
Neuritis óptica & $\varnothing$ & $\varnothing$ & 11 \\
Enfermedad inflamatoria & $\varnothing$
\end{tabular}

$\mathrm{n}=$ número de pacientes; $\mathrm{m}$ = media DE = desviación estándard; $\mathrm{r}$ = rango.

no en ausencia de neuropatía óptica compresiva distiroidea. El número de pacientes con HTO o glaucoma, así como el número medio, desviación estándar (DE), y rango de fármacos hipotensores utilizados se resumen en la tabla II.

Las variables que se incluyeron en el estudio fueron: edad, sexo, diagnóstico y tratamiento de glaucoma, tratamiento con esteroides sistémicos, tipo de operación, PIO pre y postoperatoria en posición primaria de mirada (PPM) y posición en mirada hacia arriba (PMA).

\section{Métodos estadísticos}

Todos los datos fueron introducidos en Microsoft Excel (Microsoft Inc., Redmond, WA, EEUU) para su análisis estadístico. Se calcularon las medias, la desviación estándar y se utilizó la t -Student pareada con doble cola, estableciéndose significación estadística para valores de $\mathrm{p}<0,05$.

\section{RESULTADOS}

\section{Descompresión orbitaria}

La media de PIO preoperatoria en este grupo fue de 17,3 (DE: 3,86, rango 12-25) mmHg en PPM y de
22,45 (DE: 6,36, rango19-39) mmHg en PMA. Después de la cirugía de descompresión la PIO media fue de 14,25 (DE: 3,43, rango 12-19) $\mathrm{mmHg}$ en PPM y de 18,2 (DE: 4,74, rango 12-33) mmHg en PMA, lo que supone un descenso de la PIO del 17,86\% en PPM y de un $18,93 \%$ en PMA ( $p<0,005)$.

También se analizaron los resultados según la indicación de la cirugía, la presencia de PIO preoperatoria $\geq 21 \mathrm{mmHg}$ y en los pacientes con glaucoma.

La indicación de cirugía de descompresión fue neuropatía óptica en tres pacientes (seis ojos) y de proptosis en siete pacientes (14 ojos). Los pacientes con neuropatía óptica mostraron un descenso de la PIO del 20,37\% (5 mmHg) en PPM, en comparación con el grupo de proptosis que fue del 13,5\% $(2,1 \mathrm{mmHg})$.

Los cuatro ojos con PIO preoperatoria $\geq 21$ mmHg tuvieron una PIO media de 23,2 (DE: 1,07) $\mathrm{mmHg}$ en PPM y una PIO postoperatoria de 13,75 (DE: 2,87$) \mathrm{mmHg}$, lo que supone un descenso en este subgrupo del $40,86 \%$.

En cuanto a los pacientes en tratamiento hipotensor por glaucoma (dos pacientes, cuatro ojos) la PIO preoperatoria media fue de 23 (DE: 2,16) mmHg en PPM y la postoperatoria de 15 (DE: 3,46) $\mathrm{mmHg}$, con un descenso del 34\% (8 $\mathrm{mmHg})$. El número de fármacos hipotensores por ojo en los pacientes tratados también se redujo (de 1,55 DE 1,09 a 1,2 DE 0,57 fármacos).

Tabla II.

\begin{tabular}{lccc}
\hline & Descompresión & Cirugía MOE & Pulsos 6-MPDN \\
\hline Número ojos analizados/ojos con HTO (\%) & $20 / 4(20 \%)$ & $10 / 4(40 \%)$ & $24 / 11(45 \%)$ \\
Ojos con diagnóstico de glaucoma (\%) & $4(20 \%)$ & 0 & 0 \\
Número de ojos en tratamiento hipotensor (\%) & $5(25 \%)$ & $1(10 \%)$ & $3(12,5 \%)$ \\
Número medio de fármacos hipotensores & $1,55 \mathrm{DE} 1,09$ & 1 & $1,25 \mathrm{DE} 0,57$ \\
$\quad$ por ojo en pacientes con tratamiento DE (rango) & $(1-3)$ & $(1-3)$ & \\
\hline \hline
\end{tabular}

MOE: Músculo extraocular; MPDN: Metilprednisolona; DE = desviación estándard. 


\section{Cirugía sobre MOE}

La PIO media preoperatoria fue de 18,9 (DE: 3,07, rango 16-24) mmHg en PPM y de 22,4 (DE: 6,14, rango 17-37) mmHg en PMA. La PIO postoperatoria media fue de 16,6 (DE: 3,50, rango 13-21) mmHg en PPM y de 18,6 (DE: 3,33, rango 14-25) mmHg en PMA. Esto supone un descenso del $12,16 \%$ en PPM y del 16,96\% en PMA ( $p<0,05)$. No encontramos diferencias estadísticamente significativas entre los dos grupos (cirugía de recto inferior y cirugía de recto medial y inferior). En los casos (cuatro ojos) con una PIO $\geq 21 \mathrm{mmHg}$ se objetivó una PIO media en PPM de 22 (DE: 1,41) mmHg y de 27,75 (DE: 6,80) mmHg en elevación. La PIO postoperatoria media en estos pacientes fue de 19 (DE: 2,82) mmHg en PPM (descenso del $13,86 \%$ ) y de 20,75 (DE: 3,68$) \mathrm{mmHg}$ (descenso de $25,22 \%)$.

\section{Pulsos de corticoides}

Se administraron pulsos a un total de 12 pacientes (24 ojos). La PIO pretratamiento media fue de 21,23 (DE: 6,42, rango13-39) mmHg en PPM y de 24,45 (DE: 8,15, rango 15-39) mmHg en PMA. La PIO media postratamiento fue de 17,33 (DE: 4,38, rango 9-22) $\mathrm{mmHg}$ en PPM, lo que supone un descenso del 18,75\% ( $p=0,0003)$. La PIO media en elevación postratamiento fue de 20,08 (DE: 4,86, rango 13-26) $\mathrm{mmHg}$, con un descenso del 17,86\% $(\mathrm{p}<0,001)$.
En el subgrupo de pacientes con una PIO $\geq 21$ mmHg (n=11), PIO media fue de 26,63 (DE: 5,62) $\mathrm{mmHg}$, y la PIO postratamiento media descendió a 19,72 (DE: 3,25) mmHg, es decir, un 25,94\% menos $(\mathrm{p}=0,0008)$. El número de fármacos hipotensores pasó de 1,25 DE 0,57 a 0,33 DE 0,57 fármacos por ojo. Los resultados se resumen en la tabla III.

\section{DISCUSIÓN}

Hace más de 80 años que se sabe que la PIO puede estar elevada en pacientes con OT (2). Numerosos trabajos han documentado además un aumento de PIO en elevación de la mirada en la OT, que es debida a una fibrosis del músculo recto inferior que bloquea la salida de humor acuoso por la vía episcleral y por la congestión orbitaria $(2,3)$.

Sin embargo, hay muy pocos trabajos en la literatura médica que estudien los cambios en la PIO con el tratamiento de la OT. Ohtsuka y cols (4) fueron los primeros en aportar datos, al describir los cambios en la PIO de 4 pacientes tratados mediante descompresión orbitaria. Danesh-Meyer y cols (5) confirmaron con una serie de casos más larga el descenso de la PIO postratamiento con descompresión orbitaria y con cirugía de estrabismo, no así con radioterapia orbitaria. Kikkawa y cols (6) han evidenciado también un descenso de la PIO con tratamiento del estrabismo mediante toxina botulínica.

Nuestro estudio demuestra que la PIO disminuye significativamente después del tratamiento con cirugía de descompresión y de estrabismo. Además,

\section{Tabla III.}

\begin{tabular}{|c|c|c|c|}
\hline & Descompresión & Estrabismo & 6-MPDN \\
\hline \multicolumn{4}{|l|}{ PIO preop, $m$ DE } \\
\hline PPM & 17,35 (DE: 3,86) & $18,9$ (DE: 3,07$)$ & 21,33 (DE: 6,42) \\
\hline PMA & 22,45 (DE: 6,36) & $22,4$ (DE: 6,14$)$ & 24,45 (DE: 8,15 ) \\
\hline \multicolumn{4}{|l|}{ PIO postop, m DE } \\
\hline PPM & 14,24 (DE: 3,43) & $16,6$ (DE: 3,50$)$ & 17,33 (DE: 4,38) \\
\hline PMA & 18,20 (DE: 4,74) & 18,6 (DE: 3,33 ) & $20,08$ (DE: 4,86$)$ \\
\hline \multicolumn{4}{|l|}{ \% Disminución } \\
\hline PPM & $17,86 \%$ & $12,16 \%$ & $18,75 \%$ \\
\hline PMA & $18,93 \%$ & $16,96 \%$ & $17,86 \%$ \\
\hline PIO preop $\geq 21 \mathrm{mmHg}$ & $\begin{array}{c}40,86 \% \\
(\mathrm{en} \mathrm{PPM})\end{array}$ & $\begin{array}{c}13,86 \% \\
\text { (en PPM) }\end{array}$ & $\begin{array}{c}25,94 \% \\
\text { (en PPM) }\end{array}$ \\
\hline Número medio de fármacos hipotensores por ojo & 1,55 DE 1,09 & 1 & 1,25 DE 0,57 \\
\hline Pre-tratamiento M DE + R & $(1-3)$ & & $(1-3)$ \\
\hline Número fármacos hipotensores & 1,2 DE 0,57 & 0 & $0,33 \mathrm{DE} 0,57$ \\
\hline Post-tratamiento M DE + R & $(0-2)$ & & $(0-1)$ \\
\hline
\end{tabular}

PPM: Posición primaria de mirada, PMA: posición en mirada hacia arriba, MPDN: metilprednisolona, M: media, r: rango; DE: desviación estándard. 
al contrario de lo que sucede con la radioterapia (5), el tratamiento con pulsos ha reducido la PIO en el subgrupo de pacientes estudiados.

Los resultados obtenidos en el grupo de descompresión orbitaria concuerdan con los de otros estudios publicados. La PIO postoperatoria es más baja, independientemente de la PIO preoperatoria (disminución del 17,86\%), pero en el subgrupo de pacientes con una $\mathrm{PIO}>21 \mathrm{mmHg}$ el descenso es más del doble $(40,86 \%)$.

En los pacientes con diagnóstico de glaucoma, el descenso medio fue de $8 \mathrm{mmHg}$ y valores de PIO se «normalizaron»y aunque se redujo el número de fármacos hipotensores, el tratamiento no se pudo eliminar por completo. Esto sugiere que hay factores que influyen en el aumento de la PIO que no son eficazmente tratados con la medicación para el glaucoma. Hay diversas teorías que intentan explicar el descenso de la PIO con la descompresión orbitaria. Riemann y Cols (7) realizaron una manometría orbitaria directa en 18 órbitas de pacientes afectos de OT y evidenciaron un aumento de la tensión tisular orbitaria y un descenso de la complianza orbitaria, lo que produciría un aumento de la presión retrobulbar. Otto y cols (8) midieron la presión retrobulbar durante la cirugía de descompresión en ocho pacientes con OT, obteniendo una presión media de $28,7 \mathrm{mmHg}$ (cuando en personas sanas se calcula que oscila entre 3-4,5 mmHg) (9). Después de la cirugía, la presión retrobulbar se redujo a 18,7 $\mathrm{mmHg}$. Esto sugiere que la HTO en pacientes con OT es producida, en parte, por un aumento de presión intraorbitaria y de la congestión venosa, que conducirían a un aumento de la presión venosa episcleral (PVE). Al disminuir la congestión orbitaria mediante la cirugía de descompresión, la PVE también disminuirá, con el consiguiente descenso de la PIO (Ecuación de Goldmann = entrada acuoso/salida acuoso + PVE) (9).

Los pacientes que fueron tratados mediante cirugía sobre MOE también mostraron una disminución significativa de la PIO. La PIO descendió 2,3 mmHg en PPM y 3,8 mmHg en elevación. Los resultados son muy parecidos a la serie de casos publicada por Danesh-Meyer y cols (5). La contracción del recto superior en elevación presionaría el globo contra un recto inferior fibrótico o inflamado, lo que produciría un aumento de la PIO en elevación. En pacientes con una marcada fibrosis del recto inferior, éste tiende a empujar el ojo hacia abajo y debe hacer fuerza en elevación para mantenerlo en posición primaria. Esto llevaría a un aumento de la PIO en posición primaria, por el mismo mecanismo que en elevación.

En cuanto a los pacientes tratados con pulsos de 6-MPDN, la PIO postratamiento descendió 3,9 mmHg en PPM y 4,37 mmHg en elevación. En los pacientes con una $\mathrm{PIO}>21 \mathrm{mmHg}$, la $\mathrm{PIO}$ disminuyó un $25 \%$. Rutecka-Debniak y cols (10) aportaron una serie de casos donde concluían que los esteroides sistémicos eran más eficaces que la radioterapia orbitaria para controlar pacientes con PIO elevada y OT. Danesh-Meyer y cols (5) concluyeron en su trabajo que la radioterapia orbitaria no disminuyó significativamente la PIO. En la OT hay un aumento del contenido orbitario secundario a un aumento de la musculatura extraocular, depósito de glucosaminoglicanos, e infiltración linfocitaria de la grasa orbitaria. El tratamiento con corticoides disminuye el componente inflamatorio de la OT, disminuyendo el volumen orbitario. Esto podría explicar el descenso de la PIO en estos pacientes.

Así pues podemos concluir que el tratamiento de la OT mediante descompresión orbitaria (que disminuye principalmente la congestión orbitaria), cirugía de estrabismo (que disminuye la restricción de los músculos fibróticos) y los pulsos de 6MPDN (que disminuyen la inflamación orbitaria) ha producido un descenso significativo de la PIO en los 3 grupos. En los pacientes con HTO o con diagnóstico de glaucoma, se ha producido un descenso más marcado de la PIO. Así pues, creemos que después del tratamiento de la OT, el diagnóstico de glaucoma y su tratamiento deben ser reevaluados en estos pacientes. Sin embargo, son necesarios estudios prospectivos randomizados para confirmar estos resultados.

\section{BIBLIOGRAFÍA}

1. Ohtsuka K, Nakamura Y. Open-angle glaucoma associated with Graves disease. Am J Ophthalmol 2000; 129: 613-617.

2. Gamblin GT, Harper DG, Galentine P, Buck DR, Chernow B, Eil C. Prevalence of increased intraocular pressure in Graves' disease - evidence of frequent subclinical ophthalmopathy. N Engl J Med 1983; 308: 420-424.

3. Spierer A, Eisenstein Z. The role of increased intraocular pressure on upgaze in the assessment of Graves ophthalmopathy. Ophthalmology 1991; 98: 1491-1494.

4. Cockerham KP, Pal C, Jani B, Wolter A, Kennerdell JS. The prevalence and implications of ocular hypertension and glaucoma in thyroid-associated orbitopathy. Ophthalmology 1997; 104: 914-917. 
5. Ohtsuka K. Intraocular pressure and proptosis in 95 patients with Graves ophthalmopathy. Am J Ophthalmol 1997; 124: 570-572.

6. Danesh-Meyer $H V$, Savino PJ, Deramo V, Sergott $R C$, Smith AF. Intraocular pressure changes alter treatment for Graves orbitopathy. Ophthalmology 2001; 108: 145150.

7. Kikkawa DO, Cruz RC Jr, Christian WK, Rikkers $S$, Weinreb RN, Levi L, Granet DB. Botulinum A toxin injection for restrictive myopathy of thyroid-related orbitopathy: effects on intraocular pressure. Am J Ophthalmol 2003; 135: 427-431.

8. Riemann CD, Foster JA, Kosmorsky GS. Direct orbital manometry in patients with thyroid-associated orbitopathy. Ophthalmology 1999; 106: 1296-1302.
9. Otto AJ, Koornneef L, Mourits MP, Deen-van Leeuwen L. Retrobulbar pressures measured during surgical decompression of the orbit. Br J Ophthalmol 1996; 80: 10421045.

10. Simonsz HJ, Harting F, de Waal BJ, Verbeeten BW. Sideways displacement and curved path of recti eye muscles. Arch Ophthalmol 1985; 103: 124-128.

11. Kalmann R, Mourits MP. Prevalence and management of elevated intraocular pressure in patients with Graves' orbitopathy. Br J Ophthalmol 1998; 82: 754-757.

12. Rutecka-Debniak A, Krzystolik Z, Pilarska K, Krzyzanowska-Swiniarska B, Syrenicz, A, Jarema A. The influence of treatment of progressive edematous infiltrative ophthalmopathy on intraocular pressure. Klin Oczna 1999; 101: 195-200. 